\title{
Enlargeable length-structure and scalar curvatures
}

\author{
Jialong Deng ${ }^{1}$ \\ Received: 5 August 2020 / Accepted: 20 April 2021 / Published online: 19 May 2021 \\ (c) The Author(s) 2021
}

\begin{abstract}
We define enlargeable length-structures on closed topological manifolds and then show that the connected sum of a closed $n$-manifold with an enlargeable Riemannian lengthstructure with an arbitrary closed smooth manifold carries no Riemannian metrics with positive scalar curvature. We show that closed smooth manifolds with a locally CAT(0)metric which is strongly equivalent to a Riemannian metric are examples of closed manifolds with an enlargeable Riemannian length-structure. Moreover, the result is correct in arbitrary dimensions based on the main result of a recent paper by Schoen and Yau. We define the positive $M V$-scalar curvature on closed orientable topological manifolds and show the compactly enlargeable length-structures are the obstructions of its existence.
\end{abstract}

\section{Introduction}

The search for obstructions to the existence of a Riemannian metric with positive scalar curvature (PSC-metric) on a closed non-simply connected smooth manifold is an ongoing project. Based on index theory methods, Gromov and Lawson introduced enlargeability as an obstruction based on index theory in [17]. Later, they relaxed the spin assumption in dimensions less than 8 in [18, section 12]. Recently, Schoen and Yau showed that the manifold $N^{n} \# T^{n}$ carries no PSC-metrics by geometric measure theory and minimal surfaces methods in [28], where $N^{n}$ is any closed oriented smooth $n$-manifold and $T^{n}$ is a torus. Then, Cecchini and Schick used Schoen and Yau's results to show that a closed enlargeable manifold cannot carry any PSC-metric in [9].

Both enlargeabilities mentioned above are defined on Riemannian metrics and need at least $C^{1}$-smoothness for the maps. Here an enlargeable length-structure will be defined for length metric spaces and the maps only require to be continuous. Combining enlargeable length-structures with Gromov's Spherical Lipschitz Bound Theorem (SLB Theorem) [21], a new obstruction to the existence of PSC-metrics and positive $M V$-scalar curvature $S c^{M V}$ on a closed manifold is given. Details will be given later.

Theorem A Let $X^{n}(2 \leq n \leq 8)$ be a closed orientable smooth manifold, then $X^{n}$ carries no PSC-metrics in its enlargeable length-structures.

Jialong Deng

jialong.deng@ mathematik.uni-goettingen.de

1 Mathematisches Institut, Georg-August-Universität, Göttingen, Germany 
Theorem B Let $N^{n}(2 \leq n \leq 8)$ be an arbitrary closed oriented smooth $n$-manifold and $M^{n}$ be a closed manifold with an enlargeable Riemannian length-structure. Then $N^{n} \# M^{n}$ does not admit a PSC-metric.

Remark 1.1 In fact, $N^{n} \# M^{n}$ does not admit a complete uniformly PSC-metric for any oriented manifold $N^{n}(2 \leq n \leq 8)$.

Theorem C If $\left(X^{n}, d, \mathcal{H}_{d}^{n}\right)(n \leq 8)$ satisfies $S c^{M V}\left(X^{n}\right) \geq \kappa>0$, then $d$ is not in any compactly enlargeable length-structures on the closed oriented topological manifold $X$.

Based on Schoen and Yau's argument [28], Gromov proved the SLB Theorem [21, Section 3] for any dimensions. Similarly, another consequence of [28] is that theorems A and $\mathrm{B}$ are also true in higher dimensions.

Theorem B' If $N^{n}$ is an arbitrary closed oriented smooth $n$-manifold and $X^{n}$ is a closed manifold with an enlargeable Riemannian length-structure, then $N^{n} \# X^{n}$ carries no PSC-metrics.

In particular, if $M^{n}$ is a closed manifold with an enlargeable Riemannian length-structure containing a locally CAT(O)-metric, then $N^{n} \# M^{n}$ does not admit a PSC-metric.

Remark 1.2 Gromov also stated the result which said that a closed spin manifold with a locally CAT(0)-metric carries no PSC-metrics without proof in [20, Section 4.1.2]. Theorem B' supports Gromov's assertion.

Remark 1.3 Though Theorem B' is similar to Cecchini and Schick's Theorem A [9] and the starting point of the proofs is Schoen and Yau's new results [28], the techniques of the proofs are different. Cecchini and Schick's proof used the standard constructions from geometric measure theory, especially, no symmetrization and no manifolds with boundary, whereas the proof of Theorem B' is an application of Gromov's Spherical Lipschitz Bound Theorem that uses these two ingredients.

Historical remark. Theorem $\mathrm{B}^{\prime}$ is also inspired by an open conjecture that a closed aspherical manifold does not admit a PSC-metric. Kasparov and Skandalis [24] used KKtheory to prove the strong Novikov conjecture for CAT(0)-group, implying that any locally CAT(0)-manifold carries no PSC-metrics.

The fact that a closed Riemannian manifold with non-positive sectional curvature is enlargeable is well-known since Gromov and Lawson first proposed the definition of enlargeable manifolds [17]. Riemannian metrics with non-positive sectional curvature (non-positive curvature metric) are locally CAT(0)-metrics. If a manifold of dimension 2 or 3 admits a locally CAT(0)-metric, then it also admits non-positive curvature metrics, according to the classic surface theory and the Thurston-Perelmann Geometrization Theorem [13, Proposition 1].

But there is a difference between locally CAT(0)-metrics and non-positive curvature metrics in dimensions $\geq 4$. Aravinda and Farrell [4] showed that the existence of nonpositive curvature metric is not a homeomorphism invariant in general, but the existence of a locally CAT(0)-metric is homeomorphism invariant. The existence of a non-positive 
curvature metric depends on that of the smooth structure. Furthermore, locally CAT(0)manifolds which do not support a smooth structure in dimensions $\geq 5$ were constructed by Davis and Hausmann [10].

Aspherical $n$-manifolds $(n \geq 4)$ that are not covered by Euclidean space were first constructed by Davis [11]. Davis, Januszkiewicz and Lafont [13] constructed a closed smooth four-dimensional manifold $M^{4}$, which supports locally CAT(0)-metrics and whose universal cover $\widetilde{M^{4}}$ is diffeomorphic to $\mathbf{R}^{\mathbf{4}}$, but $\pi_{1}$ is not isomorphic to the fundamental group of any compact Riemannian manifold with non-positive curvature. In dimensions $\geq 5$, Davis and Januszkiewicz [12, (5b.1) Theorem] constructed a topological locally CAT(0)-manifold, whose universal cover is not homeomorphic to $\mathbf{R}^{\mathbf{n}}$. They also constructed a smooth locally CAT(0)-manifold whose universal cover is homeomorphic to $\mathbf{R}^{\mathbf{n}}$, but the boundary at infinity is distinct from $S^{n-1}$ in (5c.1) Theorem. Furthermore, those locally CAT(0)-metrics in (5c.1) Theorem are strongly equivalent to the length metrics induced by Riemannian metrics since they come from simplicial metrics for the smooth triangulations of the smooth manifold and the hyperbolization of polyhedra.

More examples of exotic aspherical manifolds can be found in [3] and [13, Section 3].

Therefore, the connect sum of a closed manifold and Davis's exotic aspherical manifold, as new examples be detected by our methods, carries no PSC-metrics.

Remark 1.4 Let $M^{n}(n \geq 5)$ be the locally CAT(0)-manifold, whose universal cover is distinct from $\mathbf{R}^{\mathbf{n}}$, as above-mentioned, and $N$ be an arbitrary closed locally CAT(0)-manifold, then the product $M^{n} \times N$ is a locally CAT(0)-manifold, which does not support any nonpositive curvature metrics. Otherwise, if $M^{n} \times N$ carries a non-positive curvature metric, then, by Lawson-Yau's splitting theorem [7], $M^{n} \times N$ is homeomorphic to $M_{1} \times N_{1}$ such that $M_{1}$ and $N_{1}$ are endowed with non-positive curvature metrics and $\pi_{1}\left(M_{1}\right)=\pi_{1}\left(M^{n}\right)$ and $\pi_{1}\left(N_{1}\right)=\pi_{1}(N)$. And then we use the proof of Borel conjecture for Riemannian manifold with non-positive sectional curvature by Farrell and Jones [15] [29] to show that $M_{1}$ is homeomorphic to $M^{n}$. Thus, $M_{1}$ also admits the locally CAT(0)-metric such that the universal cover is distinct from $\mathbf{R}^{\mathbf{n}}$. That is a contradiction. More details of the proof can be found in [13, Proposition 2].

Furthermore, using Davis's construction, Sapir [3, Corollary 1.2] firstly created closed aspherical topological $n$-manifolds $(n \geq 4)$ whose fundamental groups coarsely contain expanders. Thus, Sapir's aspherical manifolds have infinite asymptotic dimension, are not coarsely embeddable into a Hilbert space, do not satisfy G. Yu's property A, do not satisfy the Baum-Connes conjecture with coefficients. Combining Davis's construction and Sapir's techniques, Osajda constructed closed aspherical topological $n$-manifolds $(n \geq 4)$ whose fundamental groups contain coarsely embedded expanders. Therefore, Sapir's and Osajda's aspherical $n$-manifolds $(n \geq 8)$ do not admit PSC-metrics by our results.

The paper is organized as follows: In Sect. 2, we define enlargeable length-structures and prove Theorems A and B'. In Sect. 3, we define the positive $M V$-scalar curvature on closed orientable topological manifolds and show the compactly enlargeable length-structures are the obstructions of its existence. 


\section{Enlargeable length-structures, PSC-metrics and locally CAT(0)-metrics}

A metric space $(X, d)$ is a length metric space if the distance between each pair of points equals the infimum of the lengths of curves joining the points. A closed topological manifold admits length metrics [6]. Metrics induced by Riemannian metrics on a closed smooth manifold are length metrics and any two such length metrics are strongly equivalent, i.e., for any length metrics $d$ and $d^{\prime}$, there exists a $c, C>0$ such that $c d \leq d^{\prime} \leq C d$. However, unlike Riemannian metrics on compact smooth manifolds, different length metrics may not be strongly equivalent to each other. For instance, two length metrics, among which one is induced by a Riemannian metric and the other by a Finsler metric, are topologically equivalent, i.e., they induce the same topology, but there may not be strongly equivalent in general.

If $\pi: \hat{X} \rightarrow X$ is a covering map, then length metrics, being local, lift from $X$ to $\hat{X}$. Furthermore, every length metric $d$ on $X$ lifts to a unique length metric $\hat{d}$ for which the covering map is a local isometry.

A topological manifold $X$ endowed with a complete length metric is called $\varepsilon$-hyperspherical if it admits a continuous map $f$ to $S^{n}(n=\operatorname{dim}(X))$ which is constant at infinity, of nonzero degree and such that

$$
\operatorname{Lip}(f):=\sup _{\substack{a \neq b \\ a, b \in X}} \frac{d_{S^{n}}(f(a), f(b))}{d_{X}(a, b)}<\varepsilon .
$$

Here constant at infinity means that there is a compact subset such that $f$ maps the complement of the compact subset to a point in $S^{n}$ and $S^{n}$ is endowed with standard round metric. From now on, $S^{n}$ is also a length metric space induced by the standard round metric $d_{S^{n}}$.

Definition 2.1 (Enlargeable length-structures) A length metric $d$ on a closed orientable n-dimensional topological manifold $X^{n}$ is said to be enlargeable if for each $\varepsilon>0$ there is an oriented covering manifold $\widetilde{X^{n}}$ endowed with the induced metric $\widetilde{d}$ that is $\varepsilon$-hyperspherical.

An enlargeable length-structure on $X^{n}$ is a strongly equivalent class of an enlargeable metric.

An enlargeable Riemannian length-structure on a closed orientable smooth manifold is an enlargeable length-structure that contains a length metric induced by a Riemannian metric on the manifold.

The length metric induced by a Riemannian flat metric on $T^{n}$ is an enlargeable metric and $T^{n}$ endowed with this enlargeable length-structure is an important example of the manifolds with an enlargeable length-structure. Enlargeable length-structures can also be defined on a closed non-orientable manifold by lifting the metric onto the orientation cover.

Remark 2.2 The enlargeable length-structure may be used to deal with positive scalar curvature in the metric geometry setting. For instance, the definition of scalar curvature for length metrics was given in [30].

Besides, the existence of a PSC-metric depends on the smooth structure. Trying to use length structure to study the PSC-metrics in Riemannian geometry, we define the enlargeable Riemannian length-structure. 
Question 2.3 Is there a closed topological manifold with two different enlargeable lengthstructures? In particular, is there an enlargeable length-structure on closed smooth manifold such that the manifold does not carry an enlargeable Riemannian length-structure?

Remark 2.4 Following Lemma 2.11 shows that a locally CAT(0)-metric is enlargeable only on the assumption that it is strongly equivalent to a Riemannian metric. Especially, the example from the introduction of a non-smoothable manifold with a locally CAT(0)-metric is not known to be enlargeable.

Property 2.5 (Properties of the enlargeable metric)

(1) Let $\left(X, d_{X}\right)$ and $\left(Y, d_{Y}\right)$ be closed orientable manifolds with length metrics and suppose there exists a Lipschitz continuous map $F:\left(X, d_{X}\right) \rightarrow\left(Y, d_{Y}\right)$ of nonzero degree. If $d_{Y}$ is an enlargeable metric on $Y$, then so is $d_{X}$.

(2) The product of two enlargeable metrics is an enlargeable metric.

Proof Suppose that $\widetilde{Y} \rightarrow Y$ is a covering space and $\widetilde{Y}$ is $\varepsilon$-hyperspherical, i.e., it admits a continuous map $f: \widetilde{Y} \rightarrow S^{n}(n=\operatorname{dim}(Y))$ that is constant at infinity, of nonzero degree and such that $\operatorname{Lip}(f)<\varepsilon$. Let $\widetilde{X} \rightarrow X$ be the covering corresponding to the subgroup $F_{*}^{-1}\left(\pi_{1}(\widetilde{Y})\right)$ and $\widetilde{X}$ be endowed with the induced metric $\widetilde{d}_{X}$, then $F$ can be lifted to a proper map $\widetilde{F}: \widetilde{X} \rightarrow \widetilde{Y}$, which is still a continuous map with Lipschitz constant $\operatorname{Lip}(F)$. It implies the map $f \circ \widetilde{F}$ is constant at infinity, $\operatorname{Lip}(f \circ \widetilde{F})<\operatorname{Lip}(F) \varepsilon$ and of nonzero degree. Thus $d_{X}$ is an enlargeable metric on $X$.

The fact that the composed map

$$
S^{n} \times S^{m} \rightarrow S^{n} \wedge S^{m} \rightarrow S^{n+m}
$$

is Lipschitz continuity and nonzero degree implies (2).

The following two corollaries are immediate consequences of Property 2.5 .

Corollary 2.6 If a closed manifold $X$ carries an enlargeable length-structure, then $X \times S^{1}$ still carries an enlargeable length-structure.

Corollary 2.7 Let $\left(X, d_{X}\right)$ be a closed smooth manifold, where $d_{X}$ is induced by a Riemannian metric, and $\left(Y, d_{Y}\right)$ be another manifold, where $d_{Y}$ is in the enlargeable length-structure. Suppose there exists a Lipschitz continuous map $F:\left(X, d_{X}\right) \rightarrow\left(Y, d_{Y}\right)$ of nonzero degree, then $X$ carries an enlargeable Riemannian length-structure.

Theorem 2.8 (SLB Theorem [21]) If the scalar curvature of a (possibly incomplete) Riemannian $n$-manifold $X^{n}(2 \leq n \leq 8)$ is bounded from below by $n(n-1)$, then for all continuous maps $f$ from $X^{n}$ to the sphere $S^{n}$ that are constant at infinity and of nonzero degree, they hold that $\operatorname{Lip}(f)>\frac{C}{\sqrt{n} \pi}$. Here $S^{n}$ is endowed with the standard round metric and $C>\frac{1}{3}$.

Question 2.9 Let $\left(N^{n}, g\right)$ be a complete Riemannian $n$-manifold (compact or non-compact) with scalar curvature bounded below by $n(n-1)$. Let $f$ be a continuous map from $N^{n}$ to the sphere $S^{n}$ with standard round metric of nonzero degree that is constant at infinity. Is $\operatorname{Lip}(f)$ bounded from blow by one? 
Remark 2.10 A positive answer to Question 2.9 would allow us to also cover Llarull's rigidity theorems [26, Theorem A and B], Lohkamp's results [27], and their remarkable corollaries.

Gromov aimed to improve the lower bound of the Lipschitz constant in [21, Section 3] and discussed that the Lipschitz bound would be 1 . Here, the existence of a uniformly positive lower bound of the Lipschitz constant is used as the main tool to detect the obstruction to the existence of PSC-metrics.

Proof of Theorem $A$ Assume $X^{n}$ carries an enlargeable length-structure, there exists a length metric $d$ such that one orientable covering $\left(\widetilde{X^{n}}, \tilde{d}\right)$ is $\varepsilon$-hyperspherical ( $\varepsilon$ small enough). If $X^{n}$ admits a PSC-metric $g$ in the enlargeable length-structure, then the Lipschitz constant of all maps (maps are constant at infinity and nonzero degree) from $\left(\widetilde{X^{n}}, \widetilde{g}\right)$ to $S^{n}$ has a uniformly positive lower bound $C$ by the SLB Theorem. Besides, there are positive constants $\alpha_{1}$ and $\alpha_{2}$ such that $\alpha_{1} d \leq d_{g} \leq \alpha_{2} d$ by the definition of enlargeable length-structure, where $d_{g}$ is the induced metric by $g$ on $X^{n}$. Then, the Lipschitz constant of the map from $\left(\widetilde{X^{n}}, \tilde{d}\right)$ to $S^{n}$ has the uniformly lower bound $\alpha_{1} C$, which contradicts the $\varepsilon$-hypersphericity.

Let $\left(X, d_{X}\right)$ be a length space. A geodesic triangle $\triangle$ in $X$ with geodesic segments as its sides is said to satisfy the CAT(0)-inequality if it is slimmer than the comparison triangle in the Euclidean plane, i.e., if there is a comparison triangle $\triangle^{\prime}$ in the Euclidean plane with sides of the same length as the sides of $\triangle$ such that the distance between points on $\triangle$ is less than or equal to the distance between corresponding points on $\triangle^{\prime}$. A length metric $d$ on $X$ is said to be a locally CAT(0)-metric if every point in $X$ has a geodesically convex neighborhood, in which every geodesic triangle satisfies the CAT(0)-inequality.

A locally CAT(0)-manifold is a topological manifold endowed with a locally CAT(0)metric. Gromov generalized the classic Hadamard-Cartan theorem to locally CAT(0)manifolds [5]: the universal cover of a locally CAT(0)-manifold endowed with the induced metric is a globally CAT(0)-manifold, i.e., every two points can be connected by a unique geodesic line and every geodesic triangle on it satisfies the CAT(0)-inequality. Thus a locally CAT(0)-manifold is aspherical, i.e., its universal cover is contractible.

Lemma 2.11 A locally CAT(O)-metric which is strongly equivalent to a Riemannian metric on a closed smooth manifold is an enlargeable metric.

Proof Let $\left(M^{n}, d\right)$ be the closed $n$-dimensional smooth locally CAT(0)-manifold, then its universal cover $\left(\widetilde{M^{n}}, \tilde{d}\right)$ is a globally CAT $(0)$-manifold by Gromov's Theorem. Consider the map

$$
f_{t}: \widetilde{M^{n}} \rightarrow \widetilde{M^{n}} \quad x \rightarrow \gamma_{x}\left(t \tilde{d}\left(x, x_{0}\right)\right)
$$

where $x_{0}$ is a fixed point in $\widetilde{M^{n}}, t \in(0,1]$ and $\gamma_{x}$ is the unique geodesic segment from $x$ to $x_{0}$. It is well defined by the property of globally CAT(0)-manifolds and is a proper map such that the degree of $f_{t}$ is nonzero. By the CAT(0)-inequality applied to the geodesic triangle with endpoints $x, y$ and $x_{0}$, one gets

$$
\tilde{d}\left(f_{t}(x), f_{t}(y)\right) \leq t d_{\mathbf{R}^{2}}(x, y)=t d(x, y)
$$


for $x, y \in \widetilde{M^{n}}$. Therefore, $\operatorname{Lip}\left(f_{t}\right) \leq t$.

Let $\pi:\left(\widetilde{M^{n}}, \tilde{d}\right) \rightarrow S^{n}$ be a collapse map around $x_{0}$. Then the degree of $\pi$ is 1 and $\operatorname{Lip}(\pi) \leq C$ by the smoothness of $\pi$, the fact that $d$ is strongly equivalent to a Riemannian metric and the compactness of $M^{n}$. Thus, $\pi \circ f_{t}: \widetilde{M^{n}} \rightarrow S^{n}$ has nonzero degree and $\operatorname{Lip}\left(\pi \circ f_{t}\right) \leq t C$. For any small $\varepsilon>0$, we can choose $t$ such that $\left(\widetilde{M^{n}}, \tilde{d}\right)$ is $\varepsilon$-hyperspherical. That means that a manifold endowed with a locally CAT(0)-metric is enlargeable.

Remark 2.12 The assumption that the locally CAT(0)-metric is strongly equivalent to a Riemannian metric is used in the argument of $\operatorname{Lip}(\pi) \leq C$. It is not clear if the condition can be dropped for Lemma 2.11 .

Proof of Theorem B' Combining Lemma 2.11, Corollary 2.7, and the fact of the strong equivalence of all Riemannian metrics on a closed smooth manifold, we conclude Theorem $B^{\prime}$.

\section{$3 S c^{M V} \geq \kappa$}

To generalize the notion of PSC-metrics to non-Riemannian metric space, for example to piecewise smooth polyhedral spaces, Gromov [20, Section 5.3.1] defined the max-scalar curvature $S c^{\max }$ as follows:

Definition 3.1 (Gromov) Given a metric space $P$ which is locally compact and locally contractible, and a homology class $h \in H_{n}(P)$ defines $S c^{\max }(h)$ as the supremum of the numbers $\sigma \geq 0$, such that there exists a closed orientable Riemannian $n$-manifold $X$ with $S c(X) \geq \sigma$ and a 1-Lipschitz map $f: X \rightarrow P$, such that the fundamental homology class $[X]$ goes to $h$, $f_{*}[X]=h$. Here $S c(X)$ is the scalar curvature of $X$ and 1-Lipschitz map $f$ means $\operatorname{Lip}(f) \leq 1$.

Remark 3.2 The definition makes sense without assuming $\sigma \geq 0$. But if an $h \in H_{n}(P)$ $(n \geq 3)$ can be represented by the fundamental homology class $[X]$, then we always have $S c^{\max }(h) \geq 0$. Since a closed orientable smooth $n$-manifold $(n \geq 3)$ admits Riemannian metrics with constant negative scalar curvature by Kazdan-Warner theorem [25], then one can scale the metric such that the Lipschitz constant is small and the scalar curvature is closed to 0 .

Though $S c^{\max }([X]) \geq \inf _{x} S c(X, x)$ for all closed Riemannian manifold $X$ as observed by Gromov, the positivity of the max-scalar curvature cannot imply that it carries a PSC-metric in general. For instance, let $\Sigma$ be the exotic sphere which does not admit PSC-metrics, whose existence was showed by Hitchin in [23], and $g_{\Sigma}$ be a Riemannian metric on it. Then one can scale the round metric on the standard sphere such that the Lipschitz constant of the identity map is smaller than 1 . Thus one has $S c^{\max }([\Sigma])>0$, but $\Sigma$ does not admit PSC-metrics.

Furthermore, let $\left(N, g_{N}\right)$ be a closed oriented Riemannian manifold with a PSC-metric $g_{N}$ and $M$ be a closed oriented smooth manifold. Assume that there exists a degree one smooth map $f: N \rightarrow M$, then one gets $S c^{\max }([M]) \geq 0$ with an arbitrary smooth Riemannian metric $g$ on $M$. That means $S c^{\max }([M]) \geq 0$, even when the scalar curvature of $(M, g)$ is -1 . 
Therefore, the definition of the max-scalar curvature on a metric space needs to be improved. Gromov also proposed the $n$-volumic scalar curvature on metric measure spaces in [19, Section 26]. The $n$-volumic scalar curvature may be too general to be useful, for more discussion see [14]. The following definition is trying to mix the max-scalar curvature and $n$-volumic scalar curvature on metric measure spaces.

Let $\left(S^{2}(R), d_{S}, v l_{S}\right)$ be a Riemannian 2-sphere endowed with the round metric such that the scalar curvature equals to $2 R^{-2}$ and that $\left(\mathbf{R}^{n-2}, d_{E}, v l_{E}\right)$ is endowed with Euclidean metric, then the product manifold $S^{2}(R) \times \mathbf{R}^{n-2}$ can be endowed with the Pythagorean product metrics $d_{S \times E}:=\sqrt{d_{S}^{2}+d_{E}^{2}}$ and the volume $\operatorname{vol}_{S \times E}:=\operatorname{vol}_{S} \otimes \operatorname{vol}_{E}$.

From now on, let $X^{n}$ be a closed topological $n$-manifold and $d$ be the length metric such that the Hausdorff dimension of $\left(X^{n}, d\right)$ is $n$, i.e., $\operatorname{dim}_{H}\left(X^{n}\right)=n$. Therefore, there exists the $n$-dimension Hausdorff measure $\mu_{n}$ on it and then we normalize it by $\mathcal{H}_{d}^{n}=\mathcal{W}_{n} \mu_{n}$, where $\mathcal{W}_{n}$ is the $n$-dimensional volume (the Lebesgue measure) of a Euclidean ball of radius 1 in the $n$-dimensional Euclidean space, so that $\mathcal{H}_{d_{E}}^{n}=\operatorname{vol}_{E}$ for $\mathbf{R}^{n}$ with the Euclidean metric $d_{E}$. Therefore, we have the metric measure space $\left(X^{n}, d, \mathcal{H}_{d}^{n}\right)$ and will focus on this kind of metric measure space $\left(X^{n}, d, \mathcal{H}_{d}^{n}\right)$ in this paper.

Example 3.3 (Examples of metric measure spaces $\left(X^{n}, d, \mathcal{H}_{d}^{n}\right)$ )

1. Smooth oriented Riemannian manifolds $\left(M^{n}, g\right)$ with the induced volume forms $\left(M^{n}, d_{g}, v l_{g}\right)$ are the fundamental examples.

2. A length metric that is strongly equivalent to a Riemannian metric satisfies the requirement, since the Hausdorff dimension is bi-Lipschitz invariant.

3. Locally CAT(0)-manifolds with induced Hausdorff measure are examples, as the Hausdorff dimension of a closed topological $n$-manifold with a locally CAT(0)-metric is $n$.

4. If $X^{n}$ admits an length metric $d^{\prime}$ such that $\left(X^{n}, d^{\prime}\right)$ is a Alexandrov space with curvature bounded from below, then $\operatorname{dim}_{H}\left(X^{n}\right)=n$. Then the Alexandrov space is also an example.

Note that there is only one reasonable notion of volume for Riemannian manifolds. But one can define Finslerian volumes for Finsler metrics in different ways and obtain essentially different results [8, Proposition 5.5.12].

We will define the positive of $M V$-scalar curvature $S c^{M V}$ on the metric measure space $\left(X^{n}, d, \mathcal{H}_{d}^{n}\right)$.

Definition $3.4\left(S c^{M V} \geq \kappa\right)$ The $M V$-scalar curvature of $X^{n}$ is bounded from below by $\kappa>0$ for $X^{n}=\left(X^{n}, d, \mathcal{H}_{d}^{n}\right)$, i.e., $S c^{M V}\left(X^{n}\right) \geq \kappa>0$, if the closed oriented topological $n$-dimensional $X^{n}$ satisfies the following two conditions:

(1). The metric space $\left(X^{n}, d\right)$ satisfies $S c^{\max }\left(\left[X^{n}\right]\right) \geq \kappa$ for the fundamental class $\left[X^{n}\right] \in H_{n}\left(X^{n} ; \mathbf{Z}\right)$.

(2). The metric measure space $X^{n}$ is locally volume-wise smaller than $S^{2}(R) \times \mathbf{R}^{n-2}=\left(S^{2}(R) \times \mathbf{R}^{n-2}, d_{S \times E}, v_{S \times l_{S \times E}}\right)$ for all $R>\frac{\sqrt{2}}{\sqrt{\kappa}}$, i.e., for $R>\frac{\sqrt{2}}{\sqrt{\kappa}}$, there is an $\epsilon_{R}>0$, which depends on $R$, such that all $\epsilon_{R}$-balls in $X$ are smaller than the $\epsilon_{R}$-balls in $S^{2}(R) \times \mathbf{R}^{n-2}, \mathcal{H}_{d}^{n}\left(B_{x}\left(\epsilon_{R}\right)\right)<\operatorname{vol}_{S \times E}\left(B_{x^{\prime}}\left(\epsilon_{R}\right)\right)$, for all $x \in X$ and $x^{\prime} \in S^{2}(R) \times \mathbf{R}^{n-2}$. 
Since $S c^{\max }\left(\left[X^{n}\right]\right)=S c^{\max }\left(-\left[X^{n}\right]\right)$, the definition is independent from the chosen of the orientation. And $S c^{M V}\left(X^{n}\right) \geq \kappa$ is invariant under the isomorphic transformation. Here an isomorphic transformation means there is $f:(X, d, \mu) \rightarrow\left(X^{\prime}, d^{\prime}, \mu^{\prime}\right)$ such that $f_{*} \mu^{\prime}=\mu$ and $f$ is isometric between $d$ and $d^{\prime}$. Thus, the definition is well-defined.

Proposition 3.5 Let $g$ be a $C^{2}$-smooth Riemannian metric on a closed oriented n-manifold $M^{n}$ with induced metric measure space $\left(M^{n}, d_{g}, v_{0}\right)$, then the scalar curvature of $g$ is bounded from below by $\kappa>0$ if and only if $\operatorname{Sc}^{M V}\left(M^{n}\right) \geq \kappa$.

Proof Assume the scalar curvature of $g$ is bounded from below by $\kappa>0$, then the volume formula of a small ball,

$$
\operatorname{vol}_{g}\left(B_{x}(r)\right)=\operatorname{vol}_{E}\left(B_{r}\right)\left[1-\frac{S c_{g}(x)}{6(n+2)} r^{2}+O\left(r^{4}\right)\right]
$$

as $r \rightarrow 0$, where $B_{x}(r)$ is an $r$-ball in $M^{n}$ and $B_{r}$ is an $r$-ball in $\mathbf{R}^{n}$, implying condition (2) in the definition of $S c^{M V}\left(M^{n}\right) \geq \kappa$. And $S c^{\max }\left(\left[M^{n}\right]\right) \geq \inf _{x} S c\left(M^{n}, x\right) \geq \kappa$ implies condition (1).

On the other hand, if $S c^{M V}\left(M^{n}\right) \geq \kappa$, then $S c_{g} \geq \kappa>0$. Otherwise, assume there exist small $\epsilon>0$ such that $S c_{g} \geq \kappa-\epsilon>0$. That means there exists a point $x_{0}$ in $M^{n}$ such that $S c_{g}\left(x_{0}\right)=\kappa-\epsilon$, as $M^{n}$ is compact and the scalar curvature is a continuous function on $M^{n}$. Thus, we can find a small $r$-ball $B_{r}\left(x_{0}\right)$ such that the volume of $B_{r}\left(x_{0}\right)$ is greater than the volume of the $r$-ball in the $S^{2}(\gamma) \times \mathbf{R}^{n-2}$ for $\gamma=\sqrt{\frac{2}{\kappa-\frac{\epsilon}{2}}}$, which is a contradiction.

Remark 3.6 The existence of length metrics on $X^{n}$ with $S c^{M V}\left(X^{n}\right) \geq \kappa$ is the invariant under bi-Lipshchitz mapping. However, the positivity of the $S c^{M V}$ cannot imply that it carries a PSC-metric in general.

For instance, one can use the identity map between the exotic sphere $\Sigma^{n}$ above and the standard sphere to pull back the length metric which is induced by the standard round metric to $\Sigma^{n}$, i.e., giving id : $\Sigma^{n} \rightarrow\left(S^{n}, d_{S^{n}}\right)$ gets $\left(\Sigma^{n}, \mathrm{i} d^{*} d_{S^{n}}\right)$. Then one has $S c^{M V}\left(\Sigma^{n}\right) \geq n(n-1)$ for the metric measure space $\left(\Sigma^{n}, \mathrm{i} d^{*} d_{S^{n}}, \mathcal{H}_{\mathrm{i} d^{*} d_{S^{n}}}\right)$. But $i d^{*} d_{S^{n}}$ is not induced by any $C^{2}$ -smooth Riemannian metric on $\Sigma^{n}$.

Remark 3.7 The example of the exotic sphere above shows that the condition (1) cannot imply the condition (2) in the definition of $S c^{M V} \geq \kappa$ in general. The condition (2) also cannot imply the condition (1) in general. Since one can choose the length metric induced by a Finsler metric such that the induced Hausdorff measure is locally volume-wise smaller than $S^{2}(R) \times \mathbf{R}^{n-2}=\left(S^{2}(R) \times \mathbf{R}^{n-2}, d_{S \times E}, \operatorname{vol}_{S \times E}\right)$ for all $R>\frac{\sqrt{2}}{\sqrt{\kappa}}$, and the Finsler metric is not bi-Lipshchitz equivalent to a Riemannian metric in general.

Question 3.8 Let $N^{n}$ be a closed orientable aspherical $n$-manifold. Does there exist an orientable closed Riemannian $n$-manifold $M^{n}$ positive scalar curvature such that there exists a nonzero degree map $f$ from $M^{n}$ to $N^{n}$ ?

Remark 3.9 Question 3.8 is inspired by the conjecture that a closed aspherical manifold does not carry PSC-metrics. It is natural to ask that whether a closed aspherical manifold admits a length metric with positive max-scalar curvature or positive of $S c^{M V}$. 
Proposition 3.10 (Quadratic Scaling) Assume that $\left(X^{n}, d, \mathcal{H}_{d}^{n}\right)$ satisfies $S c^{M V}\left(X^{n}\right) \geq \kappa>0$, then $S c^{M V}\left(\lambda X^{n}\right) \geq \lambda^{-2} \kappa$ for all $\lambda>0$, where $\lambda X^{n}:=\left(X^{n}, \lambda \cdot d, \lambda^{n} \cdot \mathcal{H}_{d}^{n}\right)$.

Proof If we scale $d$ by $\lambda \neq 0$, then $\mathcal{H}_{\lambda d}^{n}=\lambda^{n} \mathcal{H}_{d}^{n}$. Combining it with the fact that the $S c\left(\lambda^{2} g\right)=\lambda^{-2} S c(g)$ for a smooth Riemannian metric will complete the proof.

Let $\pi: \hat{X}^{n} \rightarrow X^{n}$ be a covering map, then the length metric $d$ on $X^{n}$ is lifted to a unique length metric $\hat{d}$ such that the covering map is a local isometry. Hence $\operatorname{dim}_{H}(X, d)=\operatorname{dim}_{H}(\hat{X}, \hat{d})$ for a finite connected cover of $X$. Then we endow the lifting length metric on a finite cover of $\left(X^{n}, d, \mathcal{H}_{d}^{n}\right)$ such that $\left(\hat{X}^{n}, \hat{d}, \mathcal{H}_{\hat{d}}^{n}\right)$ is a metric measure space.

Proposition 3.11 Assume $\left(X^{n}, d, \mathcal{H}_{d}^{n}\right)$ satisfies $S c^{M V}\left(X^{n}\right) \geq \kappa>0$ and $\hat{X}^{n}$ is a finite connected cover of $X^{n}$, then $S c^{M V}\left(\hat{X}^{n}\right) \geq \kappa$ for $\left(\hat{X}^{n}, \hat{d}, \mathcal{H}_{\hat{d}}^{n}\right)$.

Proof As $\left(\hat{X}^{n}, \hat{d}, \mathcal{H}_{\hat{d}}^{n}\right)$ is locally isometric to $\left(X^{n}, d, \mathcal{H}_{d}^{n}\right)$ and $S c^{M V}\left(X^{n}\right) \geq \kappa>0,\left(\hat{X}^{n}, \hat{d}, \mathcal{H}_{\hat{d}}^{n}\right)$ is also locally volume-wise smaller than $S^{2}(R) \times \mathbf{R}^{n-2}$.

Let $\left(M^{n}, g\right)$ be the closed orientable Riemannian manifold with $S c(g) \geq \kappa$ such that $f: M^{n} \rightarrow X^{n}$ is 1-Lipschitz map and $f_{*}\left(\left[M^{n}\right]\right)=\left[X^{n}\right]$. Then $f^{*} \hat{X}^{n}$ is a finite cover of $M^{n}$ and we denote it by $\hat{M}^{n}$, i.e., $\hat{M}^{n}:=f^{*} \hat{X}^{n}$. Then the Lipschitz constant of $\hat{f}:\left(\hat{M}^{n}, \hat{g}\right) \rightarrow\left(\hat{X}^{n}, \hat{d}\right)$ is 1 , where $\hat{g}$ is the lifting metric of $g$. Then we have the following two commutative diagrams.

Using the wrong-way map, we can map $\left[X^{n}\right]$ to $H_{n}\left(\hat{X}^{n} ; \mathbf{Z}\right)$, denoting it by $\left[\hat{X}^{n}\right]$ and then we choose $\left[\hat{X}^{n}\right]$ as the fundamental class of $\hat{X}^{n}$. Again, we map $\left[\hat{X}^{n}\right]$ to $H_{n}\left(\hat{M}^{n} ; \mathbf{Z}\right)$, denoting it by $\left[\hat{M}^{n}\right]$ and choose $\left[\hat{M}^{n}\right]$ as the fundamental class of $\hat{M}^{n}$, i.e., $\hat{f}_{*}\left(\left[\hat{M}^{n}\right]\right)=\left[\hat{X}^{n}\right]$.

Thus, $S c^{M V}\left(\hat{X}^{n}\right) \geq \kappa$.

Remark 3.12 One can also define $S c^{M V} \geq \kappa>0$ for closed non-orientable topological manifolds by requiring that the double cover with the induced metric satisfies $S c^{M V} \geq \kappa>0$, since the Hausdorff dimensions are equal to each other in this case.

Proposition 3.13 (Weak SLB Theorem) Assume $\left(X^{n}, d, \mathcal{H}_{d}^{n}\right) \quad(n \leq 8)$ satisfies $S c^{M V}\left(X^{n}\right) \geq \kappa>0$, then for all continuous nonzero degree maps $h$ from $X^{n}$ to the sphere $S^{n}$, it holds that $\operatorname{Lip}(h)>\frac{C \sqrt{n-1}}{\sqrt{\kappa} \pi}$. Here $S^{n}$ is endowed with the standard round metric $d_{S^{n}}$ and $C>\frac{1}{3}$.

Proof Given a small $\epsilon>0$, there exists a closed orientable Riemannian $n$-manifold $\left(M^{n}, g\right)$ with $S c(g) \geq \kappa-\epsilon>0$ and a 1-Lipschitz and degree one map $f:\left(M^{n}, d_{g}\right) \rightarrow\left(X^{n}, d\right)$ by the definition of max-scalar curvature. Let $h$ be a continuous nonzero degree map $h:\left(X^{n}, d\right) \rightarrow\left(S^{n}, d_{S^{n}}\right)$, then

\section{$\operatorname{Lip}(h \circ f) \leq \operatorname{Lip}(h) \times \operatorname{Lip}(f)$}

induces $\operatorname{Lip}(h) \geq \operatorname{Lip}(h \circ f)$.

If one scalars the metrics $d_{g}$ and $d$ by a constant $\lambda \neq 0$, then one has $S c\left(\lambda^{2} g\right)=\lambda^{-2} S c(g), d_{\lambda^{2} g}=\lambda d_{g}$. The new maps are denoted by $\tilde{h}:\left(X^{n}, \lambda d\right) \rightarrow\left(S^{n}, d_{S^{n}}\right)$ and $\tilde{f}:\left(M^{n}, \lambda d_{g}\right) \rightarrow\left(X^{n}, d\right)$, then one has $\operatorname{Lip}(\tilde{f})=\operatorname{Lip}(f), \operatorname{Lip}(\tilde{h} \circ \tilde{f})=\lambda^{-1} \operatorname{Lip}(h \circ f)$ and $\operatorname{Lip}(\tilde{h})=\lambda^{-1} \operatorname{Lip}(h)$. 
Choose $\lambda=\sqrt{\frac{n(n-1)}{\kappa-\epsilon}}$ such that $S c\left(\lambda^{2} g\right) \geq n(n-1)$, then SLB Theorem implies that $\operatorname{Lip}(\tilde{h} \circ \tilde{f})>\frac{C}{\sqrt{n} \pi}$. That means

$$
\lambda^{-1} \operatorname{Lip}(h)=\operatorname{Lip}(\tilde{h}) \geq \operatorname{Lip}(\tilde{h} \circ \tilde{f})>\frac{C}{\sqrt{n} \pi} .
$$

Thus, we have $\operatorname{Lip}(h)>\frac{C \sqrt{n-1}}{\sqrt{\kappa-\epsilon} \pi}$. Let $\epsilon$ goes to 0 , then one has $\operatorname{Lip}(h)>\frac{C \sqrt{n-1}}{\sqrt{\kappa} \pi}$.

Gromov and Lawson define the enlargeability by allowing only finite coverings in [17] and Hanke and Schick [22] showed that the Rosenberg index of this kind of enlargeable spin manifold does not vanish, which also implies that the manifold carries no PSC-metrics.

Definition 3.14 (Compactly enlargeable length-structures) A length metric $d$ on a closed orientable n-dimensional topological manifold $X^{n}$ is said to be compactly enlargeable if for each $\varepsilon>0$ there is a finite connected covering manifold $\hat{X}^{n}$ endowed with the induced metric $\hat{d}$ which is $\varepsilon$-hyperspherical. (This notion is defined at the beginning of Section 2.)

A compactly enlargeable length-structure on $X^{n}$ is the strongly equivalent class of an compactly enlargeable metric.

A compactly enlargeable Riemannian length-structure on a closed orientable smooth manifold is an enlargeable length-structure, which contains a length metric induced by a Riemannian metric on the manifold.

Let us recall Theorem $\mathrm{C}$ and give a proof of it.

Theorem C If $\left(X^{n}, d, \mathcal{H}_{d}^{n}\right)(n \leq 8)$ satisfies $S c^{M V}\left(X^{n}\right) \geq \kappa>0$, then $d$ is not in any compactly enlargeable length-structures on the closed oriented topological manifold $X$.

Proof The argument is the same in the proof of Theorem A . Assume $d$ is in a compactly enlargeable length-structure, then there exists a compactly enlargeable metric $d^{\prime}$ on $X^{n}$ such that $\alpha_{1} d \leq d^{\prime} \leq \alpha_{2} d$ for some $0<\alpha_{1} \leq \alpha_{2}$. There exists a finite connected covering manifold $\hat{X}^{n}$ such that the induced metric $\hat{d}^{\prime}$ ) is $\varepsilon$-hyperspherical for $\varepsilon<\frac{c \sqrt{n-1}}{\sqrt{\kappa} \alpha_{2} \pi}$ by the definition of compactly enlargeable metric. That means it exists the nonzero degree map $h:\left(\hat{X}, \hat{d}^{\prime}\right) \rightarrow S^{n}$ such that $\operatorname{Lip}(h)_{\hat{d}^{\prime}}<\frac{C \sqrt{n-1}}{\sqrt{\kappa} \alpha_{2} \pi}$, where $\operatorname{Lip}(h)_{\hat{d}^{\prime}}$ is the Lipschitz constant with respect to the metric $\hat{d}^{\prime}$.

On the other hand, we have

$$
\alpha_{2}^{-1} \operatorname{Lip}(h)_{\hat{d}} \leq \operatorname{Lip}(h)_{\hat{d}^{\prime}} \leq \alpha_{1}^{-1} \operatorname{Lip}(h)_{\hat{d}} .
$$

But Proposition 3.11 shows that $S c^{M V}\left(\hat{X}^{n}, \hat{d}\right) \geq \kappa$ and then Weak SLB Theorem 3.13 shows that $\operatorname{Lip}(h)_{\hat{d}}>\frac{C \sqrt{n-1}}{\sqrt{\kappa} \pi}$, which is a contradiction.

One can also prove the proposition by Corollary 2.7 and the definition of max-scalar curvature. If $d$ is in a compactly enlargeable length-structure and $f:\left(M^{n}, g\right) \rightarrow\left(X^{n}, d\right)$ is a degree 1 map and $\operatorname{Lip}(f) \leq 1$, then $g$ is in an enlargeable Riemannian length-metric structure by Corollary 2.7. That means $M^{n}$ carries no PSC-metrics. Thus, $S c^{\max }\left(\left[X^{n}\right]\right)=0$, which is a contradiction. 
The scalar curvature of Riemannian metrics is additivity under Pythagorean-Riemannian products; however, $S c^{M V}$ may not be additivity under Pythagorean products in general. Note that for the Pythagorean product of two metric spaces $(X, d)$ and $\left(Y, d_{1}\right),\left(X \times Y, d_{2}\right)$ with $d_{2}:=\sqrt{d^{2}+d_{1}^{2}}$, we have

$$
\operatorname{dim}_{H}(X)+\operatorname{dim}_{H}(Y) \leq \operatorname{dim}_{H}(X \times Y) \leq \operatorname{dim}_{H}(X)+\operatorname{dim}_{B}(Y),
$$

where $\operatorname{dim}_{B}(Y)$ is the upper box counting dimension of $Y$, and the inequality may be strict. If $Y$ is a smooth Riemannian manifold, then $\operatorname{dim}_{H}(Y)=\operatorname{dim}_{B}(Y)$. Furthermore, we have

$$
\mathcal{H}_{d_{2}}^{n+m} \geq C(n, m) \mathcal{H}_{d}^{n} \times \mathcal{H}_{d_{1}}^{m}
$$

where $C(n, m)$ is a constant dependent only on $n$ and $m$ and $C(n, m) \geq 1 . C(n, m)$ may be greater than 1, but if $X$ and $Y$ are rectifiable Borel subsets of Euclidean space, then $C(n, m)=1$ was showed by Federer in [16, 3.2.23 Theorem].

Proposition 3.15 Assume $\left(X^{n} \times Y^{m}, d_{2}\right)$ is the Pythagorean product of $\left(X^{n}, d, \mathcal{H}_{d}^{n}\right)$ and $\left(Y^{m}, d_{1}, \mathcal{H}_{d_{1}}^{n}\right)$, where $d_{2}:=\sqrt{d^{2}+d_{1}^{2}}$, satisfies that $\operatorname{dim}_{H}\left(X^{n} \times Y^{m}\right)=n+m$ and the measure $\mathcal{H}_{d_{2}}^{n+m}:=\mathcal{H}_{d}^{n} \otimes \mathcal{H}_{d_{1}}^{m}$. Then if $S c^{M V}\left(X^{n}\right) \geq \kappa_{1}>0$ and $S c^{M V}\left(Y^{m}\right) \geq \kappa_{2}>0$, then $S c^{M V}\left(X^{n} \times Y^{m}\right) \geq \kappa_{1}+\kappa_{2}$.

Proof Since $\left(X^{n} \times Y^{m}, d_{2}\right) \quad$ is locally volume-wise smaller than $\left(S^{2}\left(R_{1}\right) \times \mathbf{R}^{n-2}\right) \times\left(S^{2}\left(R_{2}\right) \times \mathbf{R}^{m-2}\right)$ for $R_{1}>\frac{\sqrt{2}}{\sqrt{\kappa_{1}}}$ and $R_{2}>\frac{\sqrt{2}}{\sqrt{\kappa_{2}}}$, since $\mathcal{H}_{d_{2}}^{n+m}:=\mathcal{H}_{d}^{n} \otimes \mathcal{H}_{d_{1}}^{m}$. And $\left(S^{2}\left(R_{1}\right) \times \mathbf{R}^{n-2}\right) \times\left(S^{2}\left(R_{2}\right) \times \mathbf{R}^{m-2}\right) \quad$ is locally volume-wise smaller than $S^{2}\left(R_{1}+R_{2}\right) \times \mathbf{R}^{n+m-2}$ for $R_{1}+R_{2}>\frac{\sqrt{2}}{\sqrt{\kappa_{1}+\kappa_{2}}}$. Thus, we have $\left(X^{n} \times Y^{m}, d_{2}\right)$ is locally volumewise smaller than $S^{2}\left(R_{1}+R_{2}\right) \times \mathbf{R}^{n+m-2}$ for $R_{1}+R_{2}>\frac{\sqrt{2}}{\sqrt{\kappa_{1}+\kappa_{2}}}$. And one has

$$
S c^{\max }\left(\left[X^{n}\right] \otimes\left[Y^{m}\right]\right) \geq S c^{\max }\left(\left[X^{n}\right]\right)+S c^{\max }\left(\left[Y^{m}\right]\right)
$$

Hence, $S c^{\max }\left(\left[X^{n}\right] \otimes\left[M^{m}\right]\right) \geq \kappa_{1}+\kappa_{2}$.

Question 3.16 Assume that $d$ is a length metric on the closed topological $n$-manifold $X^{n}$ such that $\left(X^{n}, d\right)$ is an Alexandrov space with curvature $\geq \kappa>0$. Do we have $S c^{M V}\left(\left[X^{n}\right]\right) \geq n(n-1) \kappa ?$

Note that an Alexandrov space with curvature $\geq \kappa>0$ satisfies the Bishop-inequality [8, Theorem 10.6.8] and then it implies the condition (2) of the definition of $S c^{M V} \geq \kappa$.

Acknowledgements The author thanks Thomas Schick for the stimulating conversations, Simone Cecchini for his linguistic assistance during the preparation of this manuscript, the referee's detailed comments, and China Scholarship Council for its funding. I learned F. Thomas Farrell and his coauthor's results from his inspirational courses in Yau Center (in Tsinghua University). The note is dedicated to Professor F. Thomas Farrell's 80th birthday.

Funding Open Access funding enabled and organized by Projekt DEAL.

Open Access This article is licensed under a Creative Commons Attribution 4.0 International License, which permits use, sharing, adaptation, distribution and reproduction in any medium or format, as long as you give appropriate credit to the original author(s) and the source, provide a link to the Creative Commons licence, and indicate if changes were made. The images or other third party material in this article are included in the article's Creative Commons licence, unless indicated otherwise in a credit line to the 
material. If material is not included in the article's Creative Commons licence and your intended use is not permitted by statutory regulation or exceeds the permitted use, you will need to obtain permission directly from the copyright holder. To view a copy of this licence, visit http://creativecommons.org/licenses/by/4.0/.

\section{References}

1. Sapir, Mark: A Higman embedding preserving asphericity. J. Amer. Math. Soc. 27(1), 1-42 (2014)

2. Osajda, Damian: Small cancellation labellings of some infinite graphs and applications. Acta Math. 225(1), 159-191 (2020)

3. Ancel, F. D., Davis, M. W., Guilbault, C. R.: CAT(0) reflection manifolds. In Geometric topology (Athens, GA, 1993), vol. 2 of AMS/IP Stud. Adv. Math., pp. 441-445. Amer. Math. Soc., Providence, RI, (1997)

4. Aravinda, C.S., Farrell, F.T.: Rank 1 aspherical manifolds which do not support any nonpositively curved metric. Comm. Anal. Geom. 2(1), 65-78 (1994)

5. Ballmann, Werner, Gromov, Mikhael, Schroeder, Viktor: Manifolds of nonpositive curvature. Progress in Mathematics, vol. 61. Birkhäuser Boston Inc, Boston, MA (1985)

6. Bing, R.H.: A convex metric with unique segments. Proc. Amer. Math. Soc. 4, 167-174 (1953)

7. Blaine Lawson, H., Yau, S.T.: Compact manifolds of nonpositive curvature. J. Diff. Geo. 7, 211$228(1972)$

8. Burago, D., Burago, Y., Ivanov, S.: A course in metric geometry. Graduate Studies in Mathematics, vol. 33. American Mathematical Society, Providence, RI (2001)

9. Cecchini, S., Schick, T.: Enlargeable metrics on nonspin manifolds. Proc. Amer. Math. Soc. 149(5), 2199-2211 (2021)

10. Davis, Michael W., Hausmann, Jean-Claude: Aspherical manifolds without smooth or PL structure. In Algebraic topology (Arcata, CA, 1986), vol. 1370 of Lecture Notes in Math., pp. 135-142. Springer, Berlin, (1989)

11. Davis, M.W.: Groups generated by reflections and aspherical manifolds not covered by Euclidean space. Ann. of Math. 117(2), 293-324 (1983)

12. Davis, M.W., Januszkiewicz, T.: Hyperbolization of polyhedra. J. Differential Geom. 34(2), 347-388 (1991)

13. Davis, M., Januszkiewicz, T., Lafont, J.-F.: 4-dimensional locally CAT(0)-manifolds with no Riemannian smoothings. Duke Math. J. 161(1), 1-28 (2012)

14. Deng, J.: Curvature-dimension condition meets Gromov's $n$-volumic scalar curvature. SIGMA Symmetry Integrability Geom. Methods Appl., 17: 013, 20 pages, (2021)

15. Farrell, F. T., Jones, L. E.: Topological rigidity for compact non-positively curved manifolds. In Differential geometry: Riemannian geometry (Los Angeles, CA, 1990), Vol. 54 of Proc. Sympos. Pure Math., pp. 229-274. Amer. Math. Soc., Providence, RI, (1993)

16. Federer, H.: Geometric measure theory. Die Grundlehren der mathematischen Wissenschaften. Band 153. Springer, New York (1969)

17. Gromov, M., Lawson, H.B.: Spin and scalar curvature in the presence of a fundamental group. I. Ann. of Math. 111(2), 209-230 (1980)

18. Gromov, M.L., Blaine, H.: Positive scalar curvature and the Dirac operator on complete Riemannian manifolds. Inst. Hautes Études Sci. Publ. Math. 58, 83-196 (1984)

19. Gromov, M.: 101 Questions, Problems and Conjectures around Scalar Curvature, (2017). https://www. ihes.fr/ gromov/wp-content/uploads/2018/08/101-problemsOct1-2017.pdf

20. Gromov, M.: Four Lectures on Scalar Curvature. arXiv e-prints, page arXiv:1908.10612, Aug (2019)

21. Gromov, M.: Metric inequalities with scalar curvature. Geom. Funct. Anal. 28(3), 645-726 (2018)

22. Hanke, B., Schick, T.: Enlargeability and index theory. J. Differential Geom. 74(2), 293-320 (2006)

23. Hitchin, N.: Harmonic spinors. Adv. Math. 14, 1-55 (1974)

24. Kasparov, G., Skandalis, G.: Groups acting properly on "bolic" spaces and the Novikov conjecture. Ann. Math. 158(1), 165-206 (2003)

25. Kazdan, J.L., Warner, F.W.: Scalar curvature and conformal deformation of Riemannian structure. J. Differential Geometry 10, 113-134 (1975)

26. Llarull, M.: Sharp estimates and the Dirac operator. Math. Ann. 310(1), 55-71 (1998)

27. Lohkamp, J.: Contracting Maps and Scalar Curvature. arXiv e-prints, page arXiv:1812.11839, Dec (2018) 
28. Schoen, R., Yau, S.T.: Positive Scalar Curvature and Minimal Hypersurface Singularities. arXiv e-prints, page arXiv:1704.05490, Apr (2017)

29. Thomas F.F., Jones, L. E.: Rigidity in geometry and topology. In Proceedings of the International Congress of Mathematicians, Vol. I, II (Kyoto, 1990), pp. 653-663. Math. Soc. Japan, Tokyo, (1991)

30. Veronelli, G.: Scalar curvature via local extent. Anal. Geom. Metr. Spaces 6(1), 146-164 (2018)

Publisher's Note Springer Nature remains neutral with regard to jurisdictional claims in published maps and institutional affiliations. 